EUROPEAN JOURNAL OF PURE AND APPLIED MATHEMATICS

Vol. 13, No. 3, 2020, 414-426

ISSN 1307-5543 - www.ejpam.com

Published by New York Business Global

\title{
Existence and uniqueness of solutions for the first order non-linear differential equations with multi-point boundary conditions
}

\author{
M.J. Mardanov ${ }^{1}$, Y.A.Sharifov ${ }^{1,2, *}$, H.N. Aliyev ${ }^{3}$, R.A. Sardarova ${ }^{4}$ \\ 1 Institute of Mathematics and Mechanics, ANAS, Baku, Azerbaijan \\ 2 Baku State University Baku, Azerbaijan \\ 3 Baku Engineering University, Khirdalan city, Azerbaijan \\ 4 Azerbaijan State University of Economics (UNEC), Baku,Azerbaijan
}

\begin{abstract}
This article discusses the existence and uniqueness of solutions for the system of nonlinear first order ordinary differential equations with multipoint boundary conditions. The Green function is constructed, and the problem is reduced to the equivalent integral equation. Existence and uniqueness of the solution to this problem is studied using the Banach contraction mapping principle and Schaefer's fixed point theorem.
\end{abstract}

2020 Mathematics Subject Classifications: 34A12, 34B10, 34B15

Key Words and Phrases: Multipoint boundary conditions, existence and uniqueness solutions, fixed point theorems, first order differential equations, Schaefer's fixed point theorem.

\section{Introduction and Problem Statement}

Multipoint boundary value problems for the ordinary differential equations (ODEs) arise in modeling the broad class of natural processes. For example, if to consider the dynamical system with $n$ degrees of freedom, exactly $n$ states observed at $n$ different instants of time, then the mathematical description of this system leads to the multipoint boundary value problem. As another example we can note the vibrations of a uniform cross-section string composed of $N$ parts of different densities and also some problems in the theory of elastic stability [29]. As another example we can note the vibrations of a uniform cross-section string composed of $N$ parts of different densities and also some problems in the theory of elastic stability [29]. In some cases multipoint boundary value problems also arise when discrediting the boundary value problems for the partial differential equations. Due to these and many other strong relation with a

${ }^{*}$ Corresponding author.

DOI: https://doi.org/10.29020/nybg.ejpam.v13i3.3698

Email addresses: misirmardanov@yahoo.com (M.J. Mardanov), sharifov22@rambler.ru (Y.A.Sharifov), hualiyev@beu.edu.az (H.N. Aliyev), sardarova.rita.77@gmail.com (R.A. Sardarova) 
broad range of applications in different fields of physics and mathematics such problems are under the intensive focus of many researchers $[9,10]$.

It should be noted that the multipoint boundary value problems have been well studied for the second order differential equations (see [4, 11-13, 22, 24] and references therein). These works were mainly initiated by Ilin and Moiseev [12]. Since then, nonlinear multipoint boundary-value problems have been studied by several authors using the LeraySchauder Continuation Theorem, Leray-Schaudern nonlinear alternatives, coincidence degree theory, and fixed point theorem in cones. However, for the first order differential equations, such problems have been less studied. Examples of such works can be shown $[1,3,15-17,19,23,25,30,31]$

Similar problems for two-point and integral boundary value problems are considered in $[2,5-8,14,18,20,21,24,26-28]$.

Note that the problem under consideration in this work was also studied by M. Urabe. In [30] he gives the similar result. But those results were obtained under more strong conditions. Thus he requires the existence of the approximate solution of the considered problem with high enough accuracy that cannot be achieved in many cases. Moreover, the fundamental matrix of some quasilinear system also should be known in [30] that is difficult problem itself. The results in this work are obtained by only the initial data of the problem and we do not need solving any auxiliary problem.

In this work for the first time Green function is constructed for the multi-point boundary value problem. The considered problem is reduced to the equivalent integral equations. Then the existence and uniqueness result are studied using the Banach contraction mapping principle. The existence of the solution is also proved by applying Schaefer's fixed point theorem.

Consider the existence and uniqueness of solutions of the nonlinear differential equations of the type

$$
\dot{x}(t)=f(t, x), t \in[0, T],
$$

with multi-point boundary conditions

$$
\sum_{i=0}^{m} l_{i} x\left(t_{i}\right)=\alpha
$$

where $l_{i}, i=1,2, \ldots, m$ are constant square matrices of order $n$ such that $\operatorname{det} N \neq 0$, $N=\sum_{i=0}^{m} l_{i} ; f:[0, T] \times R^{n} \rightarrow R^{n}$ is a given function; points $t_{i}, i=1,2, \ldots, m$ satisfy the condition $0=t_{0}<t_{1}<\cdots<t_{m}=T$. We denote by $C\left([0, T] ; R^{n}\right)$ the Banach space of all continuous functions from $[0, T]$ into $R^{n}$ with the norm $\|x\|=\max \{|x(t)|: t \in[0, T]\}$ where $|\cdot|$ is the norm in the space $R^{n}$.

This paper is organized as follows. In Section 2 we introduce definitions and lemmas which are the key tools for our main result. Section 3 focuses the theorems on the existence and uniqueness of the solution of problem (1)-(2) established under some sufficient conditions on the nonlinear terms. 


\section{Preliminaries}

We define the solution of problem (1)-(2) as follows:

Definition 1. The function $x \in C\left([0, T], R^{n}\right)$ is called a solution of problem (1)-(2) if $\dot{x}(t)=f(t, x(t))$ for each $t \in[0, T]$, and boundary conditions (2) are satisfied.

For the sake of simplicity, we can consider the following problem:

$$
\begin{gathered}
\dot{x}=y(t), \quad t \in[0, T], \\
\sum_{i=0}^{m} l_{i} x\left(t_{i}\right)=\alpha .
\end{gathered}
$$

Lemma 1. Let $y \in C\left([0, T], R^{n}\right)$. Then the unique solution $x(t) \in C\left([0, T], R^{n}\right)$ of the boundary value problem for differential equation (3) with boundary conditions (4) is given by

$$
x(t)=N^{-1} \alpha+\int_{0}^{T} G(t, \tau) y(\tau) d \tau,
$$

where

$$
G(t, \tau)=\left\{\begin{array}{c}
G_{1}(t, \tau), \quad t \in\left[0, t_{1}\right], \\
G_{2}(t, \tau), \quad t \in\left(t_{1}, t_{2}\right] \\
\ldots \ldots \ldots \ldots \ldots \ldots \ldots \ldots \ldots . \\
G_{m}(t, \tau), t \in\left(t_{m-1}, T\right]
\end{array}\right.
$$

with

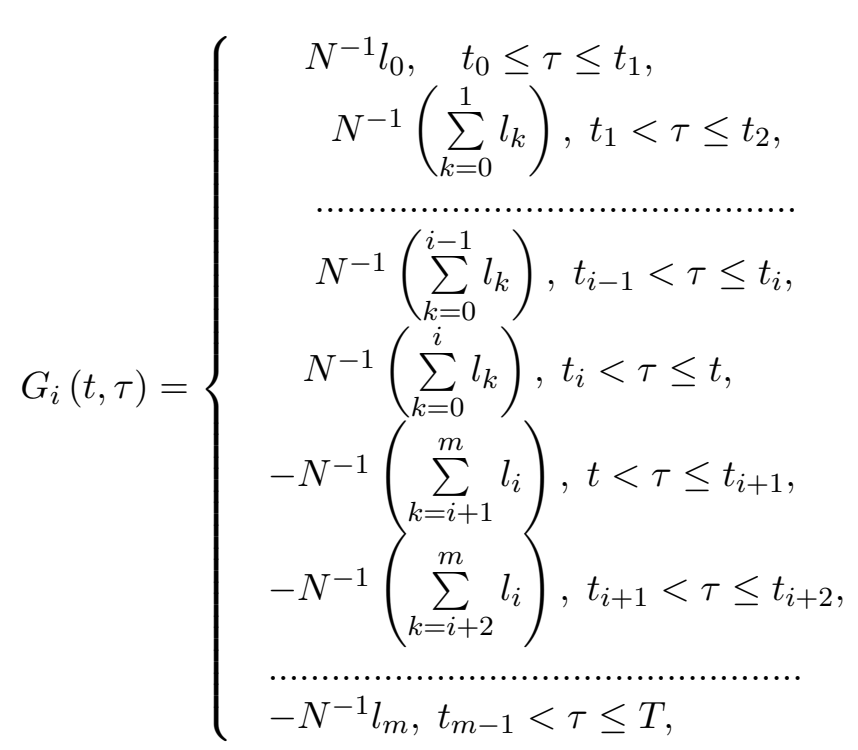

$i=1,2, \ldots, m$ 
Proof. If the function $x=x(\cdot)$ is a solution of equation (3), then for $t \in(0, T)$

$$
x(t)=x(0)+\int_{0}^{t} y(\tau) d \tau,
$$

where $x_{0}$ is an arbitrary constant vector. Now we define $x_{0}$ so that, the function in equality (6) satisfies condition (4). Then we have

$$
\sum_{i=0}^{m} l_{i}\left[x_{0}+\int_{0}^{t_{i}} y(s) d s\right]=\alpha .
$$

This obviously gives

$$
x_{0}=N^{-1} \alpha-N^{-1}\left[\sum_{i=1}^{m} l_{i} \int_{0}^{t_{i}} y(s) d s\right] .
$$

Considering the value $x_{0}$ determined from the equality (7) in (6) we get

$$
x(t)=N^{-1} \alpha-N^{-1}\left[\sum_{i=1}^{m} l_{i} \int_{0}^{t_{i}} y(s) d s\right]+\int_{0}^{t} y(s) d s .
$$

Suppose that $t \in\left[0, t_{1}\right]$ Then equality (8) may be written as follows:

$$
\begin{aligned}
& x(t)=N^{-1} \alpha-N^{-1}\left(l_{1} \int_{0}^{t} y(\tau) d \tau+l_{1} \int_{t}^{t_{1}} y(\tau) d \tau\right)-N^{-1}\left(l_{2} \int_{0}^{t} y(\tau) d \tau+l_{2} \int_{t}^{t_{1}} y(\tau) d \tau\right) \\
& -N^{-1} l_{2} \int_{t_{1}}^{t_{2}} y(\tau) d \tau-N^{-1}\left(l_{3} \int_{0}^{t} y(\tau) d \tau+l_{3} \int_{t}^{t_{1}} y(\tau) d \tau\right)-N^{-1} l_{3}\left(\sum_{i=1}^{2} \int_{t_{i}}^{t_{i+1}} y(\tau) d \tau\right) \\
& -\ldots-N^{-1}\left(l_{m} \int_{0}^{t} y(\tau) d \tau+l_{m} \int_{t}^{t_{1}} y(\tau) d \tau\right)-N^{-1} l_{m}\left(\sum_{i=1}^{m} \int_{t_{i}}^{t_{i+1}} y(\tau) d \tau\right)+\int_{0}^{t} y(\tau) d \tau .
\end{aligned}
$$

One can easily rewrite this equality in the equivalent form:

$$
\begin{gathered}
x(t)=N^{-1} \alpha+\int_{0}^{t}\left(E-N^{-1} \sum_{i=1}^{m} l_{i}\right) y(\tau) d \tau-N^{-1} \int_{t}^{t_{1}}\left(\sum_{i=1}^{m} l_{i}\right) y(\tau) d \tau \\
-N^{-1}\left(\sum_{i=2}^{m} l_{i}\right) \int_{t_{1}}^{t_{2}} y(\tau) d \tau-N^{-1}\left(\sum_{i=3}^{m} l_{i}\right) \int_{t_{2}}^{t_{3}} y(\tau) d \tau-\ldots-N^{-1} l_{m} \int_{t_{m-1}}^{T} y(\tau) d \tau,
\end{gathered}
$$


where $E$ is an identity matrix. Since equality

$$
\left(E-N^{-1} \sum_{i=1}^{m} l_{i}\right)=N^{-1} l_{0}
$$

is valid following function may be introduced

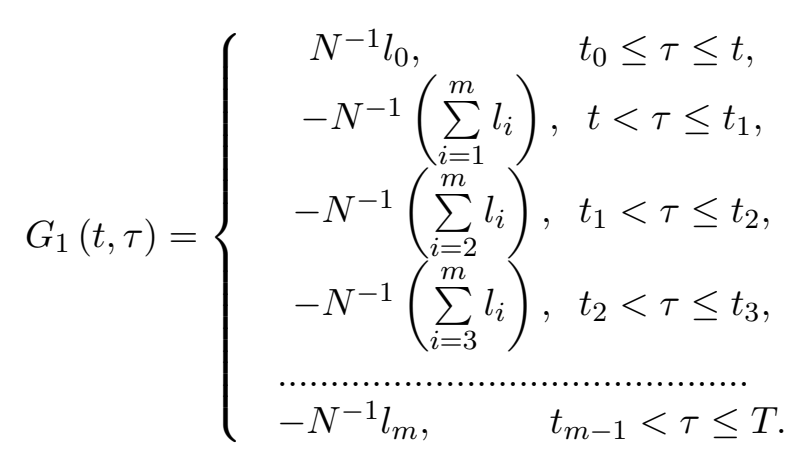

Considering the last one we can transfer equality (9) to the following an integral equation

$$
x(t)=N^{-1} \alpha+\int_{0}^{T} G_{1}(t, \tau) y(\tau) d \tau, \quad t \in\left[0, t_{1}\right] .
$$

Assuming $t \in\left(t_{1}, t_{2}\right]$ we can write equality (8) in the following form

$$
\begin{gathered}
x(t)=N^{-1} \alpha-N^{-1}\left(\sum_{i=1}^{m} l_{i}\right) \int_{0}^{t_{1}} y(t) d t-N^{-1}\left(\sum_{i=2}^{m} l_{i}\right)\left(\int_{t_{1}}^{t} y(\tau) d \tau+\int_{t}^{t_{2}} y(\tau) d \tau\right) \\
-N^{-1}\left(\sum_{i=3}^{m} l_{i}\right) \int_{t_{2}}^{t_{3}} y(\tau) d-N^{-1}\left(\sum_{i=4}^{m} l_{i}\right) \int_{t_{3}}^{t_{4}} y(\tau) d \tau-\ldots-N^{-1} l_{m} \int_{t_{m-1}}^{T} y(\tau) d \tau \\
+\int_{0}^{t_{1}} y(t) d t+\int_{t_{1}}^{t} y(\tau) d \tau .
\end{gathered}
$$

From this it is easy to derive

$$
\begin{aligned}
x(t) & =N^{-1} \alpha+N^{-1} l_{0} \int_{0}^{t_{1}} y(t) d t+N^{-1}\left(\sum_{i=0}^{1} l_{i}\right)\left(\int_{t_{1}}^{t} y(\tau) d \tau\right)-N^{-1}\left(\sum_{i=2}^{m} l\right) \int_{t}^{t_{2}} y(\tau) d \tau \\
& -N^{-1}\left(\sum_{i=3}^{m} l_{i}\right) \int_{t_{2}}^{t_{3}} y(\tau) d-N^{-1}\left(\sum_{i=4}^{m} l_{i}\right) \int_{t_{3}}^{t_{4}} y(\tau) d \tau-\ldots-N^{-1} l_{m} \int_{t_{m-1}}^{T} y(\tau) d \tau .
\end{aligned}
$$


In this step we again introduce a new function

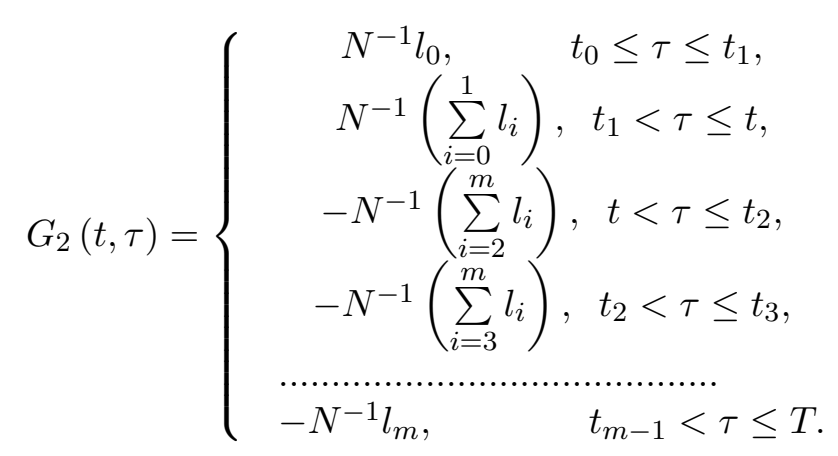

Therefore we conclude that if $t \in\left(t_{1}, t_{2}\right]$ then the solution of the considered boundary value problem can be presented in the form

$$
x(t)=N^{-1} \alpha+\int_{0}^{T} G_{2}(t, \tau) y(\tau) d \tau .
$$

Continuing this process in a similar way, for the segment $t \in\left(t_{i}, t_{i+1}\right]$ we get

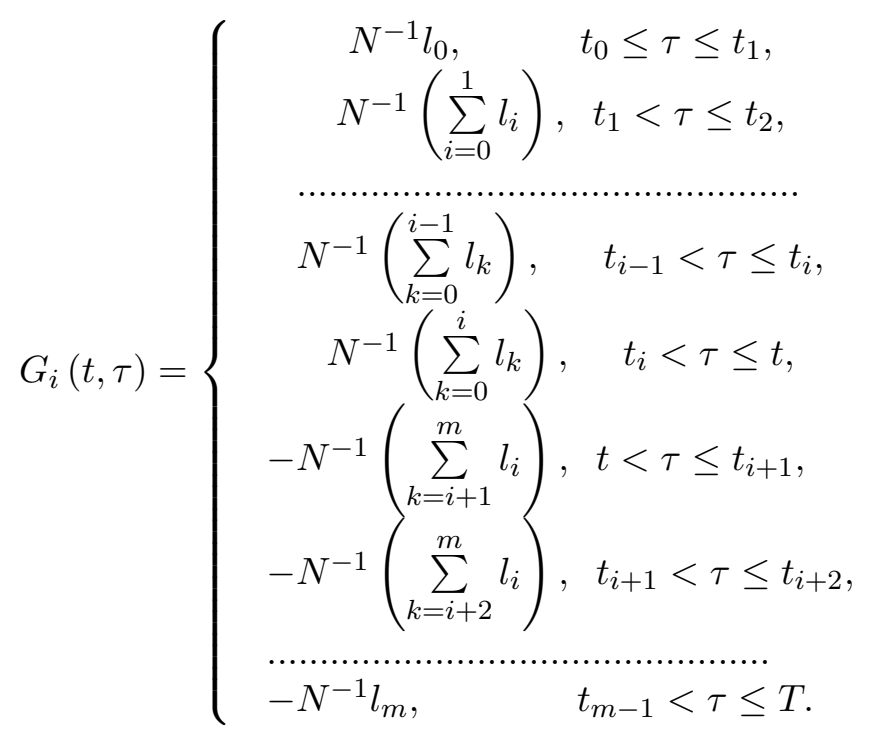

Finally we see that the solution of boundary value problem (1)-(2) may be presented in the form

$$
x(t)=N^{-1} \alpha+\int_{0}^{T} G(t, \tau) y(\tau) d \tau .
$$

Proof is completed.

Lemma 2. Let $f \in C\left([0, T] \times R^{n} ; R^{n}\right)$. Then the function $x(t)$ is a solution of boundary value problem (1)-(2) if and only if $x(t)$ is a solution of the integral equation 


$$
x(t)=N^{-1} \alpha+\int_{0}^{T} G(t, \tau) f(\tau, x(\tau)) d \tau .
$$

Proof. Let $x(t)$ be a solution of boundary value problem (1)-(2). This lemma can be proved analogously to Lemma 1 . By direct checking it is easy to justify that the solution of integral equation (10) satisfies also boundary value problem (1)-(2). Lemma 2 is proved.

\section{Main results}

Let us set the following conditions:

(H1) The function $f \in C\left([0, T] \times R^{n} ; R^{n}\right)$ is continuous;

(H2) There exist a constant $M \geq 0$ such that

$$
|f(t, x)-f(t, y)| \leq M|x-y|
$$

for $t \in[0, T]$ each and all $x, y \in R^{n}$;

(H3) There exists a constant $K \geq 0$ such that $|f(t, x)| \leq K$ for each $t \in[0, T]$ and all $x \in R^{n}$.

We give here the following uniqueness result.

Theorem 1. Assume that, assumptions(H1) and (H2) hold and

$$
L=T S M<1,
$$

where

$$
S=\max _{[0, T] \times[0, T]}\|G(t, \tau)\| .
$$

Then boundary value problem (1)-(2) has a unique solution on $[0, T]$.

Proof. To prove the statement of the above theorem we transform the boundary value problem (1)- (2) into a fixed point problem. Consider the operator

$$
(F x)(t)=N^{-1} \alpha+\int_{0}^{T} G(t, \tau) f(\tau, x(\tau)) d \tau .
$$

It is not difficult to see that

$$
F: C\left([0, T] ; R^{n}\right) \rightarrow C\left([0, T] ; R^{n}\right)
$$

Obviously, the fixed points of the operator $F$ are solutions of boundary problem (1)-(2).

Setting

$$
\max _{[0, T]}|f(t, 0)|=M_{f}
$$


we take

$$
r \geq \frac{\left\|N^{-1} d\right\|+M_{f} T S}{1-L}
$$

We show that $F B_{r} \subset B_{r}$, where

$$
B_{r}=\left\{x \in C\left([0, T] R^{n}\right):\|x\| \leq r\right\}
$$

For $x \in B_{r}$, using (H1), we get

$$
\begin{gathered}
\|(F x)(t)\| \leq\left\|N^{-1} \alpha\right\|+\int_{0}^{T}|G(t, \tau)|(|f(\tau, x(\tau))-f(\tau, 0)|+|f(\tau, 0)|) d \tau \\
\leq\left\|N^{-1} d\right\|+S \int_{0}^{T}\left(M|x|+M_{f}\right) d t \leq\left\|N^{-1} d\right\|+S M r T+M_{f} T S \leq \frac{\left\|N^{-1} \alpha\right\|+M_{f} T S}{1-L} \leq r .
\end{gathered}
$$

In order to show that the operator $F$ is a contraction, for any $x, y \in B_{r}$ we have

$$
\begin{aligned}
|F x-F y| \leq & \int_{0}^{T} \mid G(t, \tau)\left(f(\tau, x(\tau))-f(\tau, y(\tau))\left|d \tau \leq \int_{0}^{T}\right| G(t, \tau)|| f(\tau, x(\tau))-f(\tau, y(\tau)) \mid d \tau\right. \\
& \leq S M \int_{0}^{T}|x(t)-y(t)| d t \leq S M T \max _{[0, T]}|x(t)-y(t)| \leq S M T\|x-y\|
\end{aligned}
$$

or

$$
\|F x-F y\| \leq L\|x-y\| .
$$

As one can see $F$ is contraction by condition (11). So, boundary value problem (1)- (2) has a unique solution.

Now we give a theorem on the existence of solutions for the considered problem.

Theorem 2. Assume conditions(H1) and (H3) hold. Then boundary value problem (1)(2) has at least one solution on $[0, T]$.

Proof. Let $F$ be the operator defined by (12). We use Schaefer's fixed point theorem to prove that $F$ has a fixed point.

First we show that $F$ is continuous. To do this suppose that be $\left\{x_{n}\right\}$ a sequence such that $x_{n} \rightarrow x$ in $C\left([0, T] ; R^{n}\right)$. Then for each $t \in[0, T]$

$$
\begin{gathered}
\left|(F x)(t)-\left(F x_{n}\right)(t)\right|=\left|\int_{0}^{T} G(t, \tau)\left(f(\tau, x(\tau))-f\left(\tau, x_{n}(\tau)\right)\right) d \tau\right| \\
\leq T S M\left|x(t)-x_{n}(t)\right| \leq L\left\|x-x_{n}\right\| .
\end{gathered}
$$


It gives $\left\|(F x)(t)-\left(F x_{n}\right)(t)\right\| \rightarrow 0$ as $n \rightarrow \infty$, which implies that the operator $F$ is continuous.

The next step is to show that $F$ maps bounded sets from $C\left([0, T] ; R^{n}\right)$ into bounded sets in $C\left([0, T] ; R^{n}\right)$. To do this it is enough to show that for any $\eta>0$ there exists a positive constant $\omega$ such that for each $x \in B_{\eta}=\left\{x \in C\left([0, T] ; R^{n}\right):\|x\| \leq \eta\right\}$ we have $\|F(x)\| \leq \omega$. For each $t \in[0, T]$ we have

$$
|(F x)(t)| \leq\left|N^{-1} \alpha\right| \mid+T S K .
$$

From this we obtain

$$
\|(F x)(t)\| \leq\left\|N^{-1} \alpha\right\|+T S K=\omega .
$$

Now we show that $F$ maps bounded sets from $C\left([0, T] ; R^{n}\right)$ into equicontinuous sets in $C\left([0, T] ; R^{n}\right)$. Take $\xi_{1}, \xi_{2} \in[0, T], \xi_{1}<\xi_{2}$, and assume that $B_{\eta}$ is a bounded set in $C\left([0, T] ; R^{n}\right)$ and let $x \in B_{\eta}$.

Here two cases should be considered

Case 1. Let $\xi_{1}, \xi_{2} \in\left[t_{i}, t_{i}+1\right]$. Then

$$
\begin{gathered}
F\left(x\left(\xi_{2}\right)\right)-F\left(x\left(\xi_{1}\right)\right)=\int_{t_{i}}^{\xi_{2}} N^{-1}\left(\sum_{k=0}^{i} l_{i}\right) f(\tau, x(\tau)) d \tau \\
-\int_{\xi_{2}}^{t_{i+1}} N^{-1}\left(\sum_{k=i+1}^{m} l_{i}\right) f(\tau, x(\tau)) d \tau-\int_{t_{i}}^{\xi_{1}} N^{-1}\left(\sum_{k=0}^{i} l_{i}\right) f(\tau, x(\tau)) d \tau \\
+\int_{\xi_{1}}^{t_{i+1}} N^{-1}\left(\sum_{k=i+1}^{m} l_{i}\right) f(\tau, x(\tau)) d \tau=\int_{\xi_{1}}^{\xi_{2}} N^{-1}\left(\sum_{k=0}^{i} l_{i}\right) f(\tau, x(\tau)) d \tau \\
+\int_{\xi_{1}}^{\xi_{2}} N^{-1}\left(\sum_{k=i+1}^{m} l_{i}\right) f(\tau, x(\tau)) d \tau=\int_{\xi_{1}}^{\xi_{2}} f(\tau, x(\tau)) d \tau .
\end{gathered}
$$

Case 2. In this case let $\xi_{1} \in\left[t_{i-1}, t_{i}\right), \xi_{2} \in\left[t_{i}, t_{i+1}\right]$. Then

$$
\begin{gathered}
F\left(x\left(\xi_{2}\right)\right)-F\left(x\left(\xi_{1}\right)\right)=\int_{t_{i-1}}^{t_{i}} N^{-1}\left(\sum_{k=0}^{i-1} l_{i}\right) f(\tau, x(\tau)) d \tau \\
+\int_{t_{i}}^{\xi_{2}} N^{-1}\left(\sum_{k=0}^{i} l_{i}\right) f(\tau, x(\tau)) d \tau-\int_{\xi_{2}}^{t_{i+1}} N^{-1}\left(\sum_{k=i+1}^{m} l_{i}\right) f(\tau, x(\tau)) d \tau \\
-\int_{t_{i-1}}^{\xi_{1}} N^{-1}\left(\sum_{k=0}^{i-1} l_{i}\right) f(\tau, x(\tau)) d \tau+\int_{\xi_{1}}^{t_{i}} N^{-1}\left(\sum_{k=i}^{m} l_{i}\right) f(\tau, x(\tau)) d \tau
\end{gathered}
$$




$$
\begin{gathered}
+\int_{t_{i}}^{t_{i+1}} N^{-1}\left(\sum_{k=i+1}^{m} l_{i}\right) f(\tau, x(\tau)) d \tau=\int_{\xi_{1}}^{t_{i}} f(\tau, x(\tau)) d \tau \\
+\int_{t_{i}}^{\xi_{2}} f(\tau, x(\tau)) d \tau=\int_{\xi_{1}}^{\xi_{2}} f(\tau, x(\tau)) d \tau .
\end{gathered}
$$

As $t_{2} \rightarrow t_{1}$, the right-hand side of both above equalities tends to zero. Considering the above results and the Arzela-Ascoli theorem, we can conclude that $F: C\left([0, T] ; R^{n}\right) \rightarrow$ $C\left([0, T] ; R^{n}\right)$ is completely continuous.

Here we establish apriori bounds i.e. we show that the set $\Delta=\left\{x \in C\left([0, T] ; R^{n}\right)\right.$ : $x=\lambda F(x)\}$ for some $0<\lambda<1$ is bounded. Let $x \in \Delta$. Then $x=\lambda F(x)$ for some $0<\lambda<1$. Thus, for each $t \in[0, T]$ we have

$$
x(t)=\lambda N^{-1} \alpha+\lambda \int_{0}^{T} G(t, \tau) f(\tau, x(\tau)) d \tau .
$$

From here

$$
\|x\| \leq\left\|N^{-1} \alpha\right\|+S K T .
$$

Therefore, the set $\Delta$ is bounded. The statement of the Schaefer's fixed point theorem may be applied and derived that the operator $F$ has at least one fixed point. So, there exists at least one solution for problems (1)- (2) on $[0, T]$.

\section{Conclusion}

It should be noted that the method considered in this paper are general enough and can be transformed to the different forms to cover a wide class of problems. We established here the results on the existence and uniqueness of the solutions for the first order nonlinear differential equations with multi-point boundary conditions. Given in the paper method can be used in similar multi-point problems for the ordinary differential equations

$$
\dot{x}=f(t, x), t \in[0, T],
$$

with multi-point and integral boundary conditions of the form

$$
\sum_{i=0}^{m} l_{i} x\left(t_{i}\right)+\int_{0}^{T} n(t) x(t) d t=\alpha .
$$

Here $0=t_{0}<t_{1}<\ldots<t_{m-1}<t_{m}=T ; \quad n(t) \in R^{n \times n}$ is a given function; $l_{i} \in R^{n \times n}, i=$ $1,2, \ldots, m$ are given matrices; $\alpha \in R^{n}$ is a given vector; and

$$
\operatorname{det} N \neq 0, N=\sum_{i=0}^{m} l_{i}+\int_{0}^{T} n(t) d t .
$$




\section{References}

[1] V M Abdullayev. Numerical solution to optimal control problems with multipoint and integral conditions. Proceedings of the Institute of Mathematics and Mechanics, National Academy of Sciences of Azerbaijan, 44(2):171-186, 2018.

[2] B Ahmad, S Sivasundaram, and R A Khan. Generalized quasilinearization method for a first order differential equation with integral boundary condition. Dyn. Contin. Discrete Impuls. Syst., Ser. A Math. Anal, 12(2):289-296, 2005.

[3] K R Aida-zade. An approach for solving nonlinearly loaded problems for linear ordinary differential equations . Proceedings of the Institute of Mathematics and Mechanics, National Academy of Sciences of Azerbaijan, 44(2):338-350, 2018.

[4] A Alsaedi, M Alsulami, R P Agarwal, and B Ahmad. Some new nonlinear secondorder boundary value problems on an arbitrary domain. Advances in Difference Equations, 2018(227), 2018.

[5] A Ashyralyev and Y A Sharifov. Optimal control problem for impulsive systems with integral boundary conditions. AIP Conference Proceedings, 1470(1):12-15, 2012.

[6] A Ashyralyev and Y A Sharifov. Existence and uniqueness of solutions for nonlinear impulsive differential equations with two-point and integral boundary conditions . Advances in Difference Equations, 1470(1):8-11, 2012.

[7] A Ashyralyev and Y A Sharifov. Existence and uniqueness of solutions for nonlinear impulsive differential equations with two-point and integral boundary conditions. Advances in Difference Equations, 2013(173):1-11, 2013.

[8] A Ashyralyev and Y A Sharifov. Optimal control problems for impulsive systems with integral boundary conditions . Electron. Journal Differential Equations, 2013(80):111, 2013.

[9] J R Cannon. One-dimensional Heat Equation . Encyclopedia of Mathematics and its Applications, Addison-Wesley Publishing Company, advanced Book Program, Reading, MA, 23, 1984.

[10] J R Cannon, S P Esteva, and J VHoek. Galerkin procedure for the diffusionequation subject to the specification of mass. SIAM J. Numer. Anal., 24(3):499-515, 1987.

[11] J R Graef and D L Kong. Solutions of second order multi-point boundary value problems . Math. Proc. Camb. Phil. Soc., 145(2):489-510, 2008.

[12] V A Ilin and E I Moiseev. Nonlocal boundary value problem of the second kind for a Sturm-Liouville operator . Differential Equations, 23(8):979-987, 1987. 
[13] H Li and J Zhang. Existence of Nontrivial Solutions for Some Second-Order Multipoint Boundary Value Problems. Journal of Function Spaces, Article ID 6486135, $24(3): 8,2018$.

[14] M J Mardanov and Y A Sharifov. Existence results for first order nonlinear impulsive differential equations with nonlocal boundary conditions. AIP Conference Proceedings, 1676(1):020015, 2015.

[15] M J Mardanov, Y A Sharifov, and K E Ismayilova. Existence and uniqueness of solutions for nonlinear impulsive differential equations with three-point boundary conditions. e-Journal of Analysis and Applied Mathematics, 2018(1):21-36, 2018.

[16] M J Mardanov, Y A Sharifov, and K E Ismayilova. Existence and Uniqueness of Solutions for the First-Order Non-Linear Differential Equations with Three-Point Boundary Conditions. Filomat, 33(5):1387-1395, 2019.

[17] M J Mardanov, Y A Sharifov, K E Ismayilova, and S A Zamanova. Existence and Uniqueness of Solutions for the System of First-order Nonlinear Differential Equations with Three-point and Integral Boundary Conditions. European Journal of Pure and Applied Mathematics, 12(3):756-770, 2019.

[18] M J Mardanov, Y A Sharifov, and H H Molaei. Existence and uniqueness of solutions for first-order nonlinear differential equations with two-point and integral boundary conditions. Electronic Journal of Differential Equations, 2014(259):1-8, 2014.

[19] M J Mardanov, Y A Sharifov, R A Sardarova, and H N Aliyev. Existence and Uniqueness of Solutions for Nonlinear Impulsive Differential Equations with Three-Point and Integral Boundary Conditions. Azerbaijan Journal of Mathematics, 10(1):110-126, 2020 .

[20] M J Mardanov, Y A Sharifov, and F M Zeynalli. Existence and uniqueness of the solutions to impulsive nonlinear integro-differential equations with nonlocal boundary conditions. Proceedings of the Institute of Mathematics and Mechanics, National Academy of Sciences of Azerbaijan, 45(2):222-233, 2019.

[21] M J Mardanov, Y A Sharifov, and F M Zeynally. Existence and uniqueness of solutions for nonlinear impulsive differential equations with nonlocal boundary conditions. Vestn. Tomsk. Gos. Univ. Mat. Mekh., 60:61-72, 2019.

[22] A L M Martinez, E V Castelani, and R Hoto. Solving a second order $m$-point boundary value problem . NONLINEAR STUDIES, 26(1):15-26, 2019.

[23] K N Murty and S Sivasundaram. Existence and uniqueness of solution to three-point boundary value problems associated withnonlinear first order systems of differential equations. J. Math. Anal. Appl, 173(1):158-164, 1993. 
[24] J J Nieto and R Rodrguez-Lpez. Greens function for first-order multipoint boundary value problems and applications to the existence of solutions with constant sign. $J$. Math.Anal. Appl., 388:952-963, 2012.

[25] B Przeradzki and R Stanczy. Solvability of a Multi-Point Boundary Value Problem at Resonance. Journal of Mathematical Analysis and Applications, 264:253-261, 2001.

[26] Y A Sharifov. Optimality conditions in problems of control over systems of impulsive differential equations with nonlocal boundary conditions. Ukrainian Mathematical Journal, 64:958-970, 2012.

[27] Y A Sharifov. Optimal control of impulsive systems with nonlocal boundary conditions. Russian Mathematics, 57(2):65-72, 2013.

[28] Y.A. Sharifov, F.M. Zeynally, and S.M. Zeynally. Existence and uniqueness of solutions for nonlinear fractional differential equations with two-point boundary conditions. Advanced Mathematical Models, Applications, 3(1):54-62, 2018.

[29] S. Timoshenko. Theory of elastic stability. McGraw-Hill, New-York, 1961.

[30] M Urabe. An existence theorem for multi-point boundary value problems. Funkcialaj Ekvacioj., 9:43-60, 1966.

[31] Y Zhang and F Zhang. Multipoint boundary value problem of first order impulsive functional differential functional differential equation. Journal of Applied Mathematics and Computing, 31:267-278, 2009. 